\title{
Substrate Mapping and Ablation for Ventricular Tachycardia in Patients with Structural Heart Disease: How to Identify Ventricular Tachycardia Substrate
}

TAKESHI KITAMURA, MD, ${ }^{1-3,7}$ CLAIRE A. MARTIN, MD, PhD, ${ }^{1-4}$ KONSTANTINOS VLACHOS, MD, PhD, ${ }^{1-3}$ RUAIRIDH MARTIN, MD, ${ }^{1-3,5}$ ANTONIO FRONTERA, MD, PhD, ${ }^{1-3,6}$ MASATERU TAKIGAWA, MD, PhD, ${ }^{1-3}$ NATHANIEL THOMPSON, MD, ${ }^{1-3}$ GHASSEN CHENITI, MD, ${ }^{1-3}$ GREGOIRE MASSOUILLE, MD, ${ }^{1-3}$ ANNA LAM, MD, ${ }^{1-3}$ FELIX BOURIER, MD, ${ }^{1-3}$ JOSSELIN DUCHATEAU, MD, ${ }^{1-3}$ THOMAS PAMBRUN, MD, ${ }^{1-3}$ ARNAUD DENIS, MD, ${ }^{1-3}$ NICOLAS DERVAL, MD, ${ }^{1-3}$ MELEZE HOCINI, MD, ${ }^{1-3}$ MICHEL HAÏSSAGUERRE, MD, PhD, ${ }^{1-3}$ HUBERT COCHET, MD, PhD, ${ }^{1-3}$ PIERRE JAÏS, MD, PhD, ${ }^{1-3}$ and FRÉDÉRIC SACHER, MD, PhD ${ }^{1-3}$

${ }^{1} / H U$ Liryc, Electrophysiology and Heart Modeling Institute, Fondation Bordeaux Université, Pessac-Bordeaux, France ${ }^{2}$ Electrophysiology and Ablation Unit, Bordeaux University Hospital (CHU), Pessac, France

${ }^{3}$ Centre de recherche Cardio-Thoracique de Bordeaux, University of Bordeaux, Bordeaux, France

${ }^{4}$ Royal Papworth Hospital NHS Foundation Trust, Cambridge, UK

${ }^{5}$ Newcastle University, Newcastle-upon-Tyne, UK

${ }^{6}$ San Raffaele Hospital, Milan, Italy

${ }^{7}$ Tokyo Metropolitan Hiroo Hospital, Tokyo, Japan

\begin{abstract}
Catheter ablation for ventricular tachycardia (VT) has been increasingly used over the past two decades in patients with structural heart disease (SHD). In these individuals, a substrate mapping strategy is being more commonly applied to identify targets for VT ablation, which has been shown to be more effective versus targeting mappable VTs alone. There are a number of substrate mapping methods in existence that aim to explore potential VT isthmuses, although their success rates vary. Most of the reported electrogram-based mapping studies have been performed with ablation catheters; meanwhile, the use of multipolar mapping catheters with smaller electrodes and closer interelectrode spacing has emerged, which allows for an assessment of detailed near-field abnormal electrograms at a higher resolution. Another recent advancement has occurred in the use of imaging techniques in VT ablation, particularly in refining the substrate. The goal of this paper is to review the key developments and limitations of current mapping strategies of substrate-based VT ablation and their outcomes. In addition, we briefly summarize the role of cardiac imaging in delineating VT substrate.
\end{abstract}

KEYWORDS. Ablation, cardiac imaging, substrate, three-dimensional mapping system, ventricular tachycardia.
ISSN 2156-3977 (print) ISSN 2156-3993 (online) CC BY 4.0 license

(C) 2019 Innovations in Cardiac Rhythm Management

\section{Introduction}

Over the last decade, catheter ablation for ventricular tachycardia (VT) has been increasingly performed as an adjunctive therapy to antiarrhythmic drugs. ${ }^{1,2}$ With this came an increased understanding of the mechanisms of VT; notably, it commonly results due to scar-related reentry in patients with structural heart disease (SHD). Activation mapping and entrainment mapping are reasonable approaches to identify and target critical sites of 
the reentrant VT circuit for ablation in patients with tolerated reentrant VT. ${ }^{3-6}$ However, the majority of patients presenting for catheter ablation have unstable VT that hampers the accurate definition of the critical areas of the reentrant circuit with activation or entrainment mapping. ${ }^{7,8}$

Thus, substrate ablation is increasingly favored as a VT ablation strategy, with or without entrainment/activation mapping methods. Substrate-based approaches involve the identification of local abnormal ventricular electrograms that represent diseased areas consistent with potential isthmuses capable of supporting reentrant VT and may be followed even when VTs are not inducible or not hemodynamically tolerated. ${ }^{9,10}$ Although a combination of several approaches is commonly employed during VT ablation, a number of studies have examined with variable results whether a substrate-based ablation strategy may be superior or comparable to one guided predominantly by the activation/entrainment mapping of inducible and hemodynamically tolerated VTs. ${ }^{11-18}$ Critical in achieving more successful results with a substrate-based approach is an accurate representation of the pathologic substrate; various strategies to delineate this have been proposed to date. ${ }^{4,10,13-22}$

With the advent of three-dimensional (3D) electroanatomical mapping (EAM) systems in the $1990 \mathrm{~s}^{23}$ there has been a significant improvement in our ability to represent both anatomical and functional electrical information in a realtime model of the ventricle. Furthermore, the development of multipolar catheters facilitates precise scar detection, ${ }^{24-26}$ with potentially more successful results. ${ }^{27}$ In addition, cardiac imaging in the form of cardiac magnetic resonance (CMR) imaging or multidetector computed tomography (MDCT) may play a potentially important role in the preprocedural assessment of cardiac anatomy and myocardial substrate and the intraprocedural integration of the structural and electrophysiological VT substrate. ${ }^{28-31}$

In this paper, we review substrate mapping and ablation strategies and clinical outcomes for VTs in the setting of SHD and also discuss the importance of modalities for substrate detection in addition to those based on electrograms.

Dr. Denis and Dr. Derval have received speaking honoraria and consulting fees from Boston Scientific. Dr. Hocini, Dr. Haïssaguerre, and Dr. Jaïs have received lecture fees from Abbott and Biosense Webster. Dr. Sacher has received consulting fees and speaking honorarium from Abbott, Boston Scientific, Medtronic, and Biosense Webster. The other authors report no conflicts of interest for the published content. This research was supported by an ANR grant (IHU LIRYC grant no. ANR-10-IAHU-04).

Manuscript received July 16, 2018. Final version accepted August 20, 2018.

Address correspondence to: Takeshi Kitamura, MD, Department of Electrophysiology, Hôpital Cardiologique du Haut-Lévêque (Centre Hospitalier Universitaire de Bordeaux), Avenue de Magellan, 33604 Bordeaux-Pessac, France. Email: takektmr@gmail.com.

\section{Substrate mapping in patients with structural heart disease}

\section{Electrogram-based substrate detection}

The identification and modification of the arrhythmogenic substrate in the endocardium and/or epicardium are increasingly considered as composing a primary ablation strategy in patients with SHD. This technique was originally developed in the absence of 3D mapping ${ }^{9,32-34}$; however, the development of 3D mapping systems in the late $1990 \mathrm{~s}^{23}$ accelerated the use of electrogram-based techniques in detecting and localizing substrate reproducibly and feasibly. Furthermore, the recent use of ultra-highdensity mapping with catheters with multiple small electrodes and closer interelectrode spacing has enhanced the speed, density, resolution, and detailed near-field signal assessment of mapping acquisition, reducing interpolation and possibly improving clinical outcomes ${ }^{25-27,35-38}$ (Figure 1).

Bipolar voltage mapping to evaluate the electrogram peak-to-peak voltage is a widely accepted and frequently used technique to characterize substrate. Endocardially, a bipolar voltage amplitude of $1.5 \mathrm{mV}$ or more normally identifies healthy tissue. Additionally, normal ventricular myocardial bipolar electrograms are defined as sharp, biphasic or triphasic signals with a duration of $70 \mathrm{~ms}$ or less and / or with an amplitude-to-duration ratio of more than $0.046{ }^{9,39}$ Areas with voltages of $0.5 \mathrm{mV}$ to $1.5 \mathrm{mV}$ are often considered as border zones in the setting of a $3.5-\mathrm{mm}$ to $4-\mathrm{mm}$-tip, 1-mm ring electrode, and 2-mm interelectrode spacing mapping catheter (Table 1), ${ }^{10,32,34}$ even in the right ventricle. ${ }^{40}$ The definitions above have been validated by human pathologic data and radiologic studies. ${ }^{41-43}$ Areas with voltages of less than $0.5 \mathrm{mV}$ are generally considered as "dense scar"; however, low-amplitude abnormal electrograms are frequently observed in these areas. ${ }^{44}$ Therefore, in order to define unexcitable scar, the area should contain no visible electrograms (ideally using mapping catheters with smaller and narrower-spaced bipolar electrodes) and have no local pacing capture. ${ }^{45}$ In the epicardium, a bipolar voltage cutoff of $1 \mathrm{mV}$ or more ${ }^{46}$ or $1.5 \mathrm{mV}^{47}$ is considered normal. With regard to the right ventricle, epicardial $1.5 \mathrm{mV}$ can also be a reasonable bipolar voltage cutoff. ${ }^{40}$ The majority of VTs have critical circuits located in the scar border zone, ${ }^{48}$ which harbors abnormal electrograms $s^{9,32,33}$ [ie, fractionated potentials, ${ }^{49}$ double potentials, and late potentials (LPs), discrete and separated from the QRS by $40 \mathrm{~ms}^{50}$ ], which can be targeted by catheter ablation. However, it has been reported that a certain proportion of abnormal potentials are also located in regions with bipolar voltages of more than $1.5 \mathrm{mV}^{19,21}$ with abnormal electrograms occasionally unmasked by extrastimuli. 51,52 We have recorded at least $3 \%$ of substrate defined as local abnormal ventricular activity (LAVA) in voltage zones of more than $1.5 \mathrm{mV}$ (because of far-field signal annotation $^{53}$ (Figures 2A-2D). Moreover, Tung et al. ${ }^{54}$ found that $18 \%$ of critical VT isthmuses were within low-voltage areas during pacing from the site but 


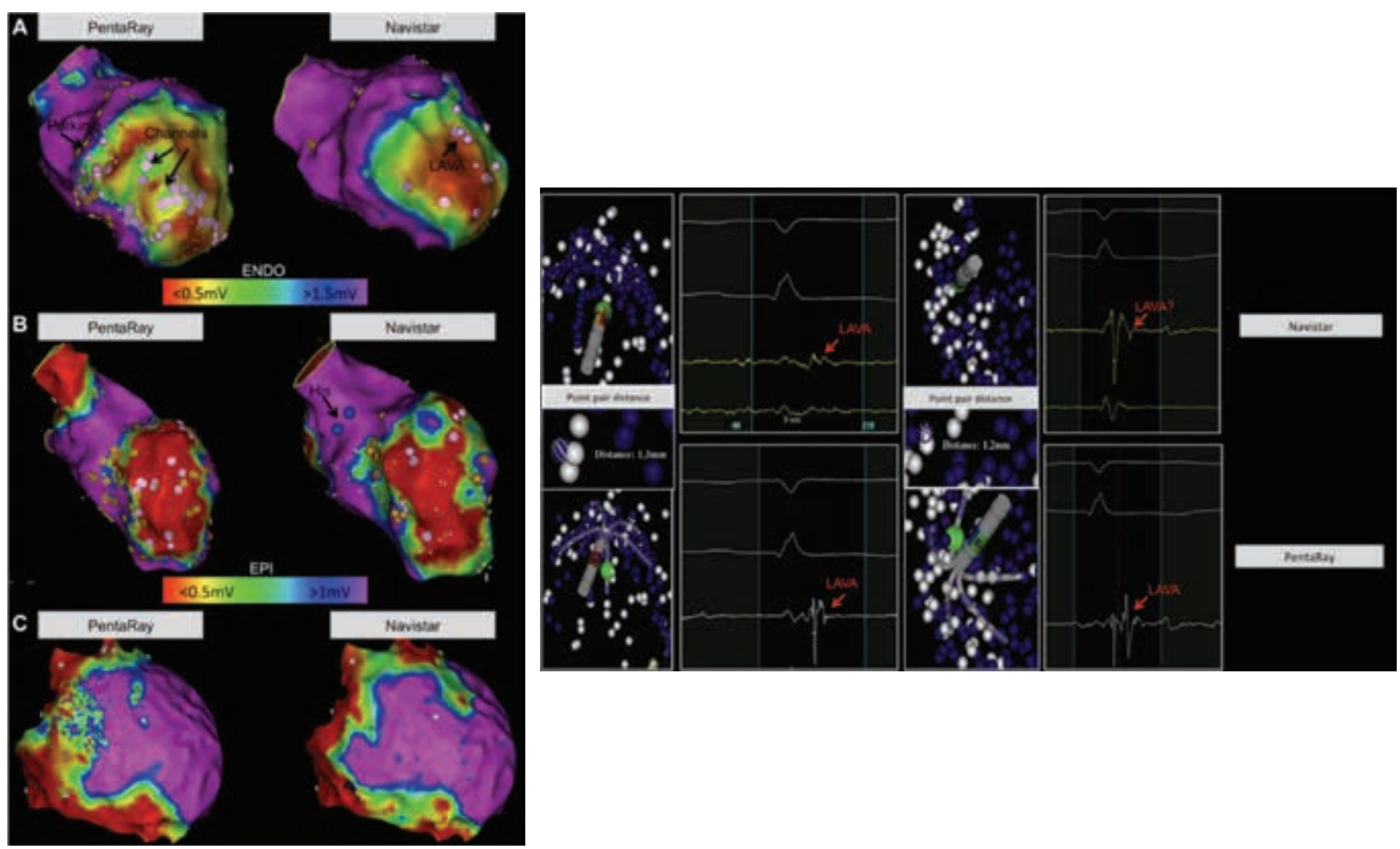

Figure 1: Left: Comparison of bipolar voltage maps (endocardial: scar $<0.5 \mathrm{mV}$, border zone $0.5-1.5 \mathrm{mV}$, and healthy tissue $>$ $1.5 \mathrm{mV}$; epicardial: border zone $0.5-1 \mathrm{mV}$ and healthy tissue $>1 \mathrm{mV}$ ) using Navistar ${ }^{\circledR}$ (Biosense Webster, Diamond Bar, CA, USA) (NAV) mapping versus PentaRay ${ }^{\circledR}$ (Biosense Webster, Diamond Bar, CA, USA) (PR) mapping in the endocardium (A) and epicardium (B) of a sheep model with an iatrogenic-created anteroseptal scar and in humans (C) using the CARTO ${ }^{\circledR} 3$ system (Biosense Webster, Diamond Bar, CA, USA). All images are shown in an anteroposterior view. A: LAVA is represented in pink, the proximal conduction (left-sided His) system is in blue, and the Purkinje system is in yellow. Border zone areas and LAVA channels are visible within the scar with PR mapping, but none are visible with NAV mapping. B: Three LAVA channels are visible with PR mapping but none with NAV mapping. The border zone is smaller and demonstrates increased detail using PR mapping. C: Larger scar area and improved border zone definition using PR versus NAV mapping. Right: Point pair analysis ( $\leq 3 \mathrm{~mm}$ of distance between a PR and NAV point) from two examples by manual signal analysis in two different patients. Substrate maps are shown at $100 \%$ transparence. LAVA using PR (in purple) and NAV (in white) are tagged. The distance between tags is measured using the distance measurement tool in the mapping system. A red arrow indicates a clear LAVA visible with PR mapping but one that is barely or not visible with NAV mapping. Reproduced with permission from: Berte B, Relan J, Sacher F, et al. Impact of electrode type on mapping of scar-related VT. J Cardiovasc Electrophysiol. 2015;26(11):1213-1223. LAVA: local abnormal ventricular activity; ENDO: endocardial; EPI: epicardial.

within normal amplitude $(>1.5 \mathrm{mV})$ areas with pacing from another site, indicating that voltage is affected by the activation wavefront. ${ }^{54-56}$ Therefore, a voltage map based on standard $(0.5-1.5 \mathrm{mV})$ voltage criteria is not necessarily capable of delineating the entire possible VT substrate.

Furthermore, with the use of high-density mapping with small electrodes and narrower interelectrode spacing (Figure 1), traditional definitions of substrate voltage need to be adjusted depending on the mapping catheter. $^{24,36}$ The electric field recorded by a pair of electrodes on a novel mapping catheter is relatively small, recording precise local signals located just underneath the electrodes. ${ }^{57,58}$ Meanwhile, endocardial unipolar voltage mapping has a large field of view and is useful to identify septal, intramural, and/or epicardial substrate, ${ }^{58-69}$ with different amplitude thresholds present depending on the etiology of the disease. The use of bipolar mapping with small electrodes and closer interelectrode spacing in combination with unipolar mapping may constitute an optimal strategy.

In summary, there are several limitations ${ }^{55}$ of conventional voltage mapping for substrate detection, with variations occurring due to the wavefront of activation, ${ }^{54-56}$ catheter interelectrode spacing, ${ }^{24,25}$ interpolation, ${ }^{63}$ far-field peak annotation of multicomponent electrograms, catheter orientation ${ }^{64}$ and contact, ${ }^{65,66}$ and surrounding insulating tissue (eg, fat, edema). Conversely, nonelectrogram techniques of substrate detection such as cardiac imaging $29,30,67-69$ are unaffected by directions of wavefront activation or techniques based on specific electrogram characterizations (see later). 


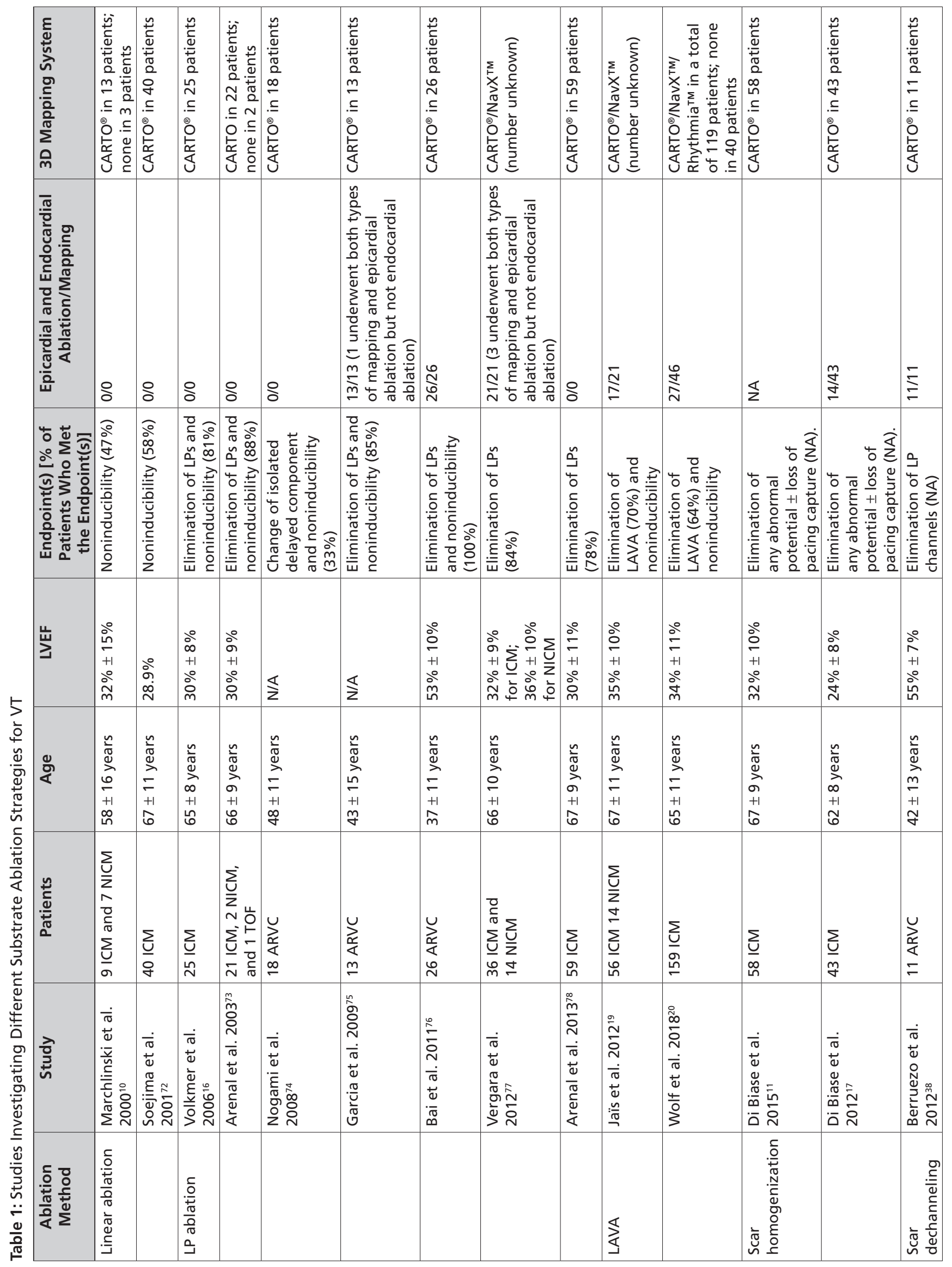




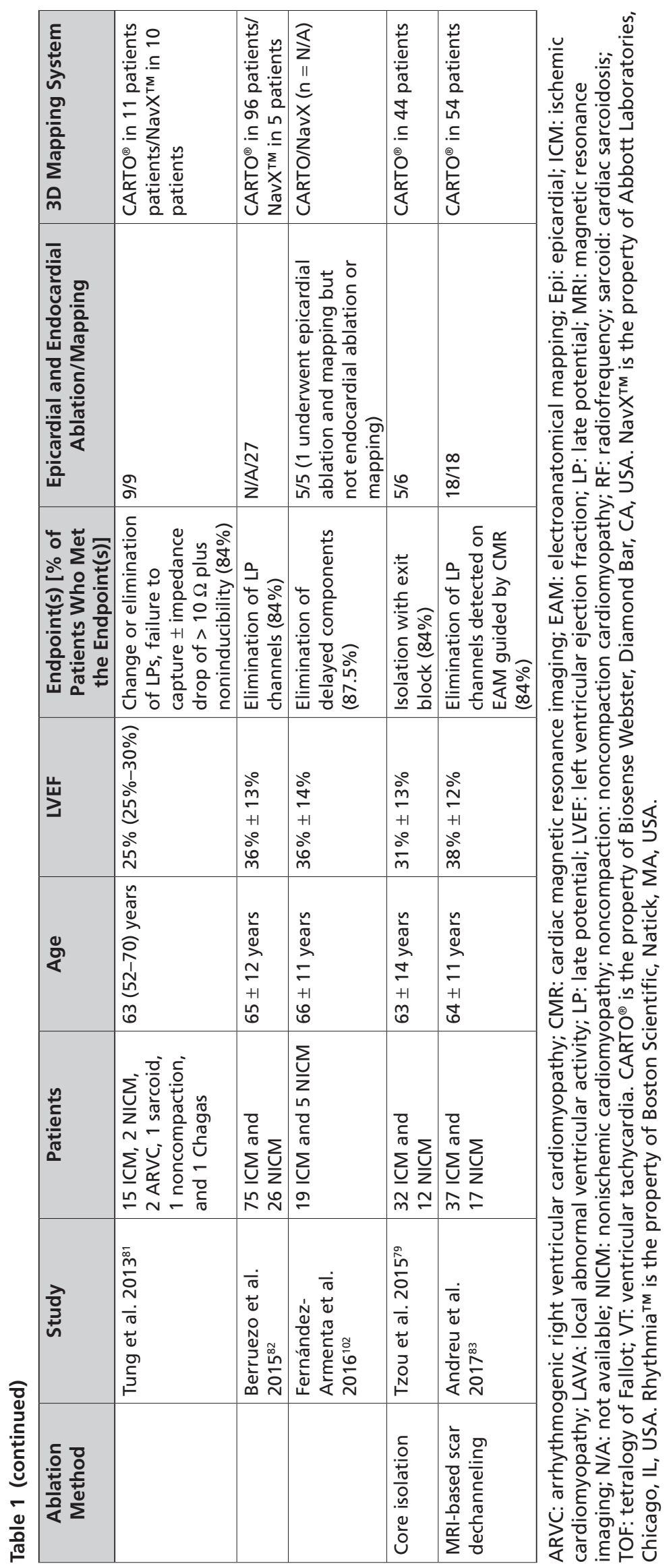




\begin{tabular}{|c|c|c|c|c|c|c|c|c|c|c|c|c|c|c|c|c|c|c|c|c|}
\hline 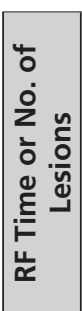 & 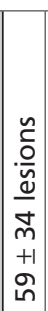 & $\begin{array}{l}\tilde{u} \\
\frac{\tilde{o}}{\tilde{n}} \\
\frac{\tilde{\omega}}{0} \\
0 \\
+1 \\
\bar{N}\end{array}$ & 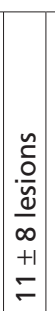 & 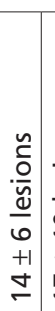 & 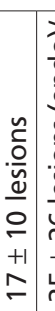 & 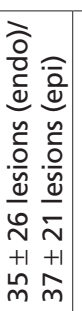 & 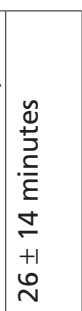 & $\frac{\$}{z}$ & 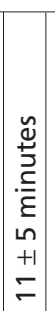 & 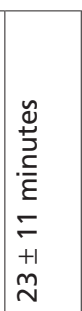 & 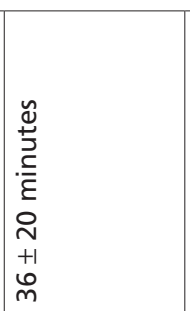 & 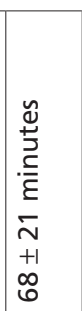 & 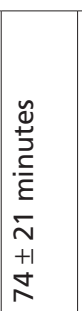 & 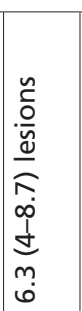 & 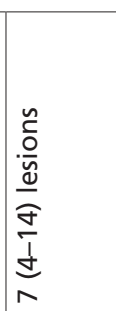 & 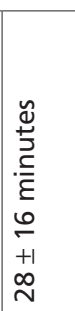 & 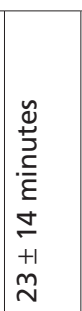 & 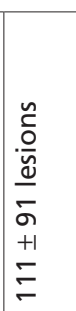 & 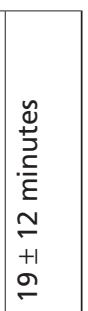 & 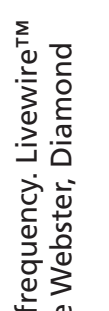 \\
\hline 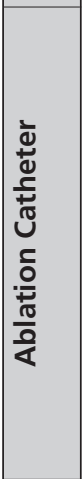 & 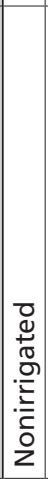 & 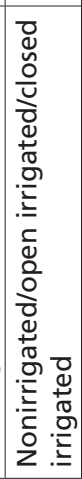 & 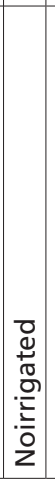 & 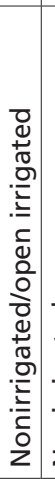 & 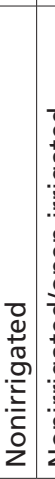 & 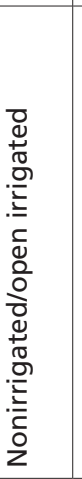 & 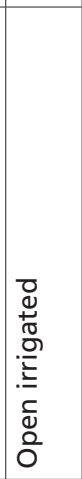 & 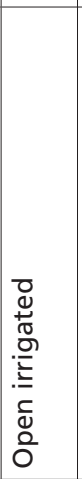 & 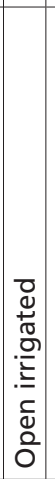 & 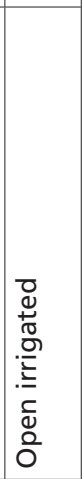 & 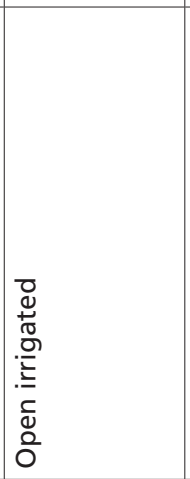 & 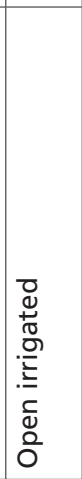 & 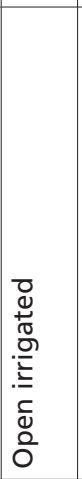 & 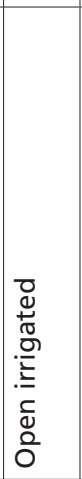 & 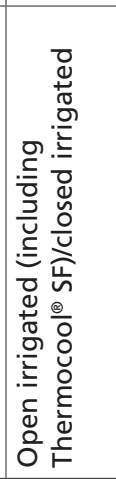 & 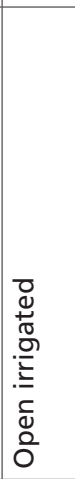 & 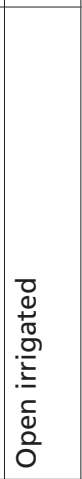 & 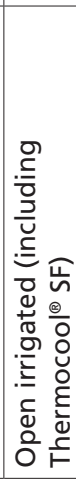 & 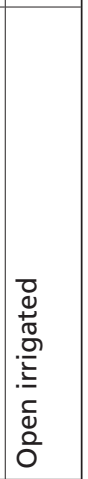 & 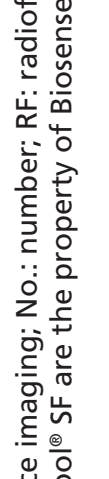 \\
\hline 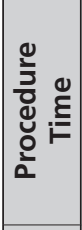 & 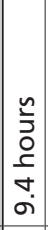 & 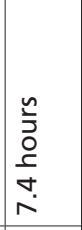 & 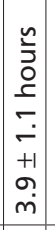 & 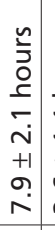 & 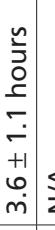 & 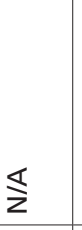 & 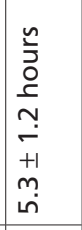 & $\Sigma$ & 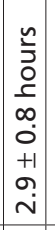 & 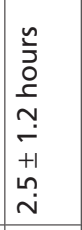 & 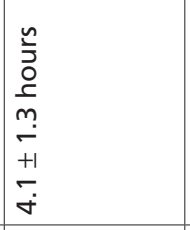 & 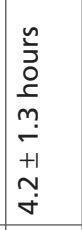 & 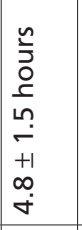 & 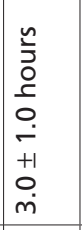 & $\frac{\nwarrow}{z}$ & 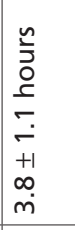 & 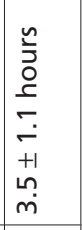 & 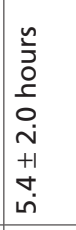 & 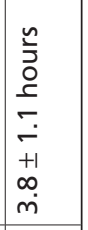 & 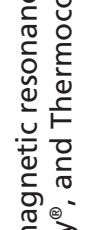 \\
\hline 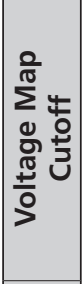 & 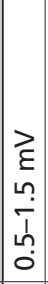 & 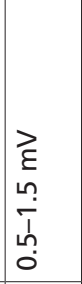 & 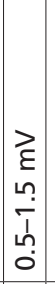 & 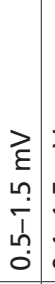 & 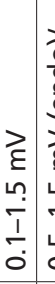 & 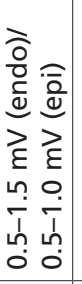 & 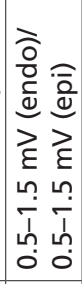 & 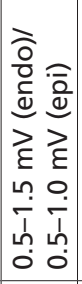 & 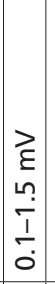 & 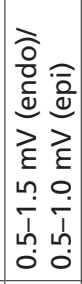 & 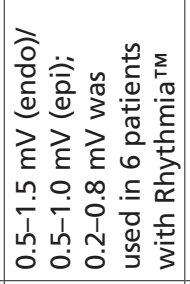 & 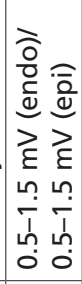 & 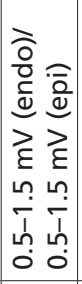 & 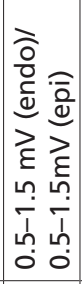 & 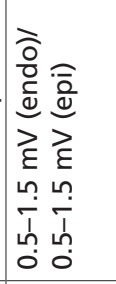 & 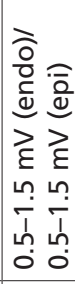 & 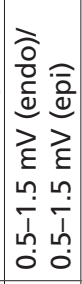 & 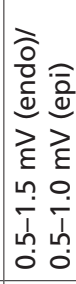 & 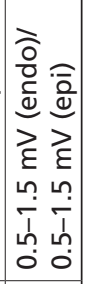 & 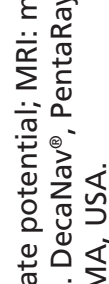 \\
\hline 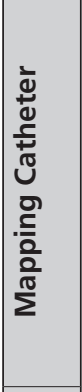 & 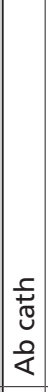 & 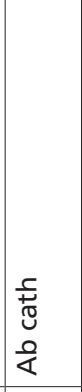 & $\mid \begin{array}{l}5 \\
\frac{5}{0} \\
0 \\
\frac{1}{4}\end{array}$ & $\begin{array}{l}\frac{5}{5} \\
0 \\
0 \\
\frac{0}{4}\end{array}$ & 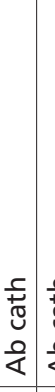 & $\begin{array}{l}\text { 莺 } \\
\text { Oे } \\
\end{array}$ & 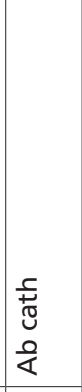 & 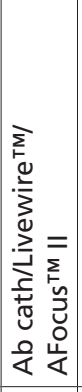 & 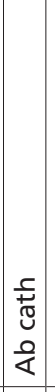 & 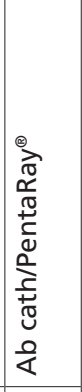 & 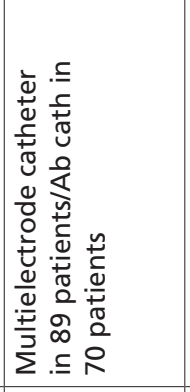 & 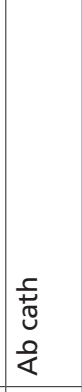 & 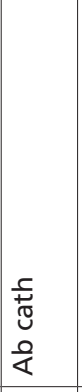 & 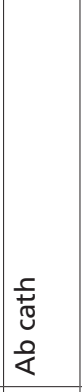 & 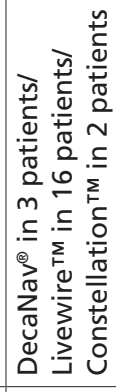 & \& & 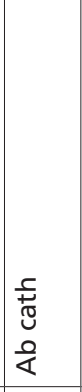 & 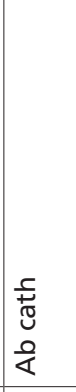 & 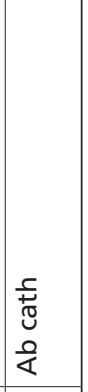 & 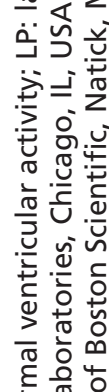 \\
\hline 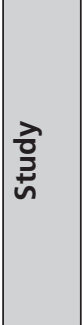 & 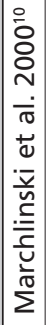 & 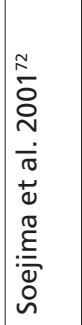 & 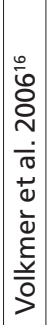 & 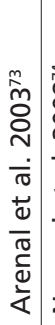 & 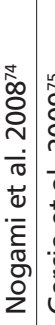 & 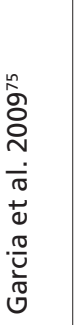 & 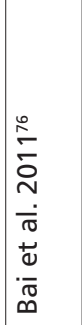 & 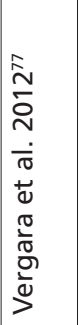 & 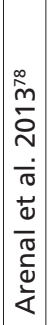 & 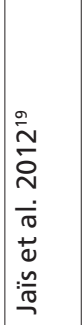 & 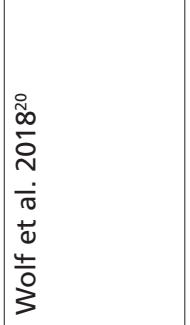 & 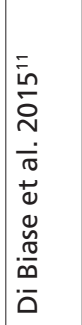 & 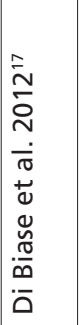 & 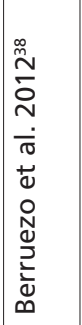 & 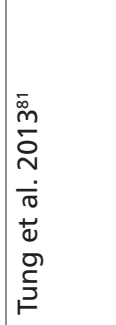 & 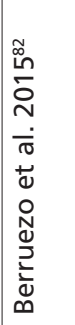 & 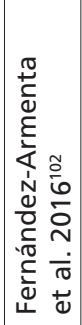 & 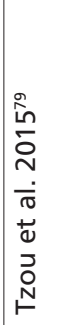 & 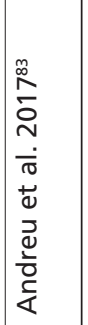 & 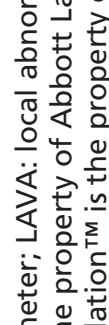 \\
\hline 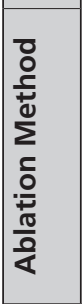 & $\mid$\begin{tabular}{l|}
$\frac{c}{0}$ \\
$\frac{0}{+0}$ \\
$\frac{0}{0}$ \\
$\frac{0}{0}$ \\
$\frac{1}{0}$ \\
\end{tabular} & & $\mid \begin{array}{l}\frac{1}{0} \\
\stackrel{0}{0} \\
\frac{\pi}{0} \\
\frac{0}{0} \\
0 \\
\end{array}$ & & & & & & & $\stackrel{5}{5}$ & & 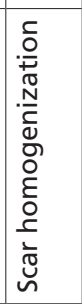 & & 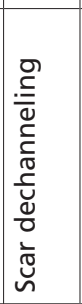 & & & & 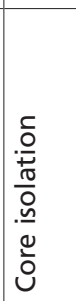 & 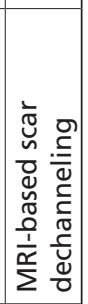 & 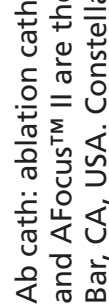 \\
\hline
\end{tabular}




\begin{tabular}{|c|c|c|c|c|c|c|c|c|c|c|c|c|c|c|c|c|c|c|c|}
\hline 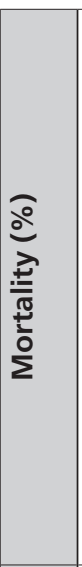 & $\leqslant$ & 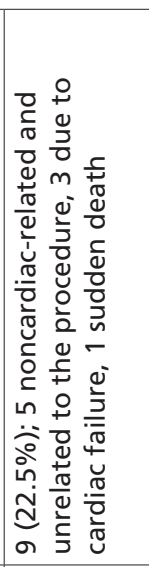 & 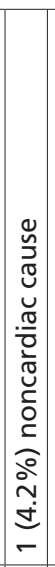 & 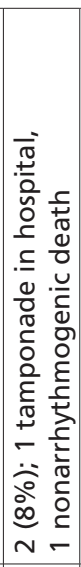 & 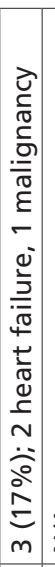 & 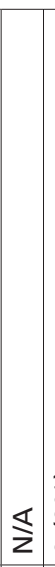 & & $\begin{array}{ccc} & \\
5 \\
\end{array}$ & 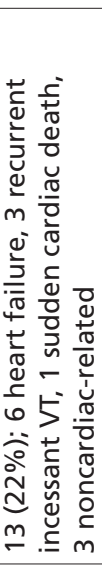 & 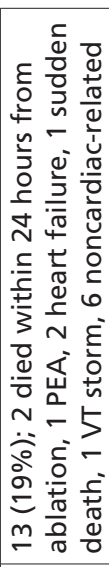 & 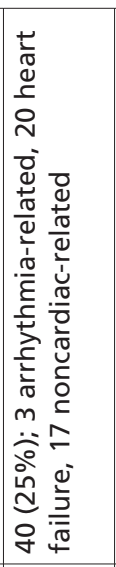 & 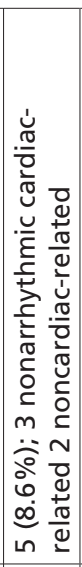 & 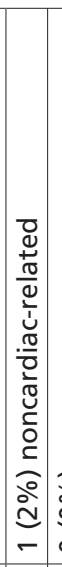 & & 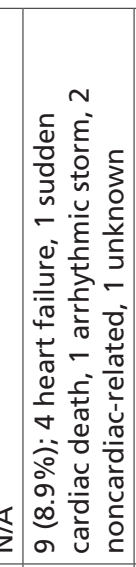 & $\frac{1}{z}$ & ôे & ఏ̊े & 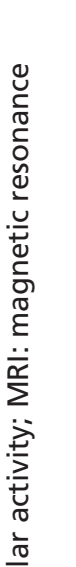 \\
\hline 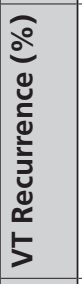 & 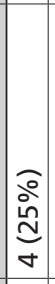 & 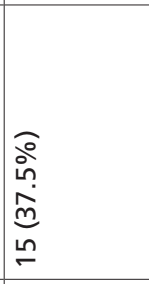 & $\mid \begin{array}{l}\widehat{a} \\
\grave{d} \\
\text { in }\end{array}$ & 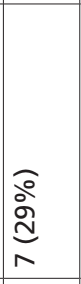 & $\begin{array}{c}0 \\
0 \\
m \\
m \\
0\end{array}$ & 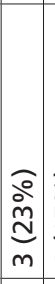 & 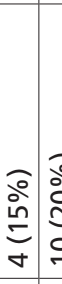 & 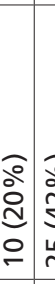 & 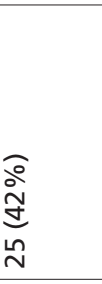 & $\begin{array}{l}\widehat{a} \\
\vdots \\
\text { do } \\
\text { m }\end{array}$ & 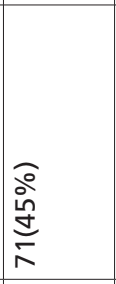 & 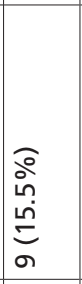 & $\begin{array}{c}0 \\
o \\
\vdots \\
\infty \\
\infty\end{array}$ & 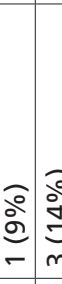 & 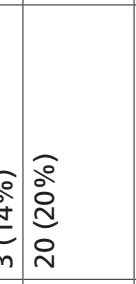 & 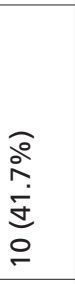 & 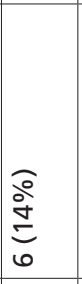 & 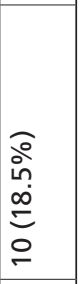 & 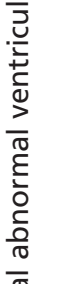 \\
\hline 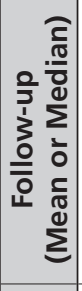 & 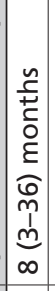 & 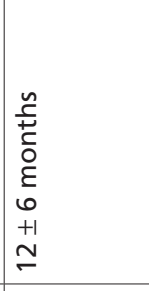 & 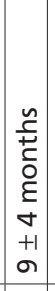 & 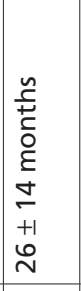 & $\begin{array}{c}u \\
+ \\
+ \\
0 \\
0 \\
\varepsilon \\
\infty \\
m \\
+1 \\
\overline{6}\end{array}$ & 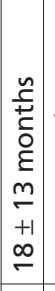 & 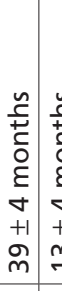 & 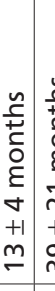 & 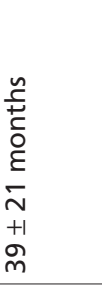 & 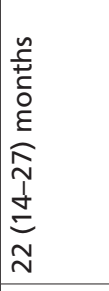 & & 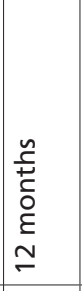 & 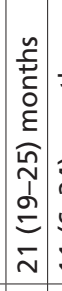 & 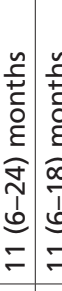 & 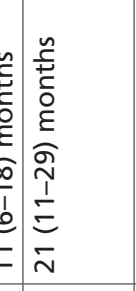 & 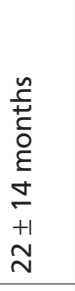 & 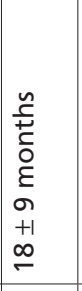 & 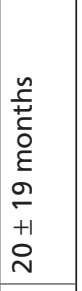 & 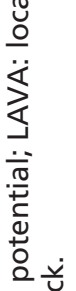 \\
\hline 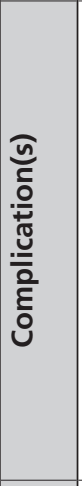 & 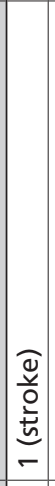 & 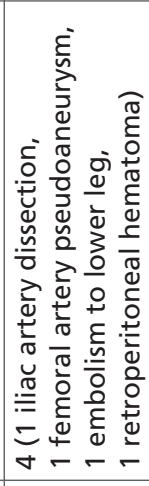 & 0 & 0 & $\frac{\$}{z}$ & 0 & 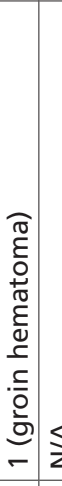 & 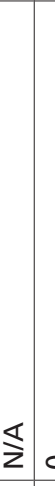 & 0 & 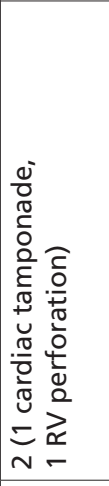 & 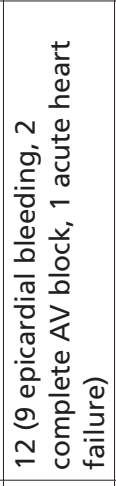 & 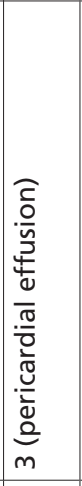 & 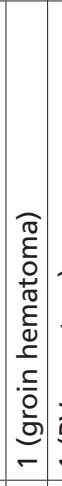 & 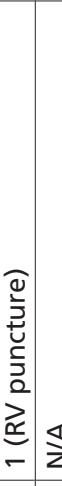 & 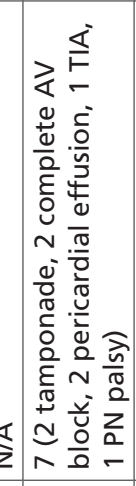 & 0 & 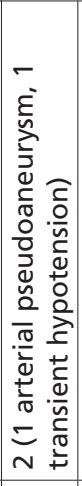 & $\frac{\pi}{z}$ & 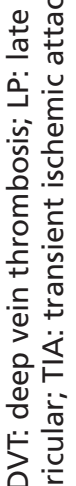 \\
\hline 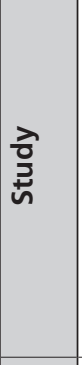 & 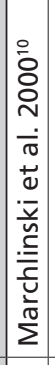 & 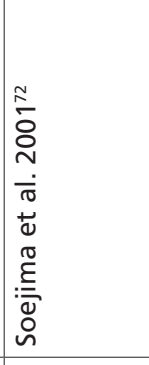 & 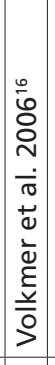 & 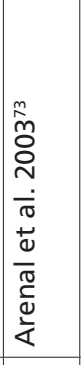 & 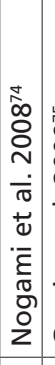 & 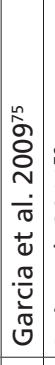 & 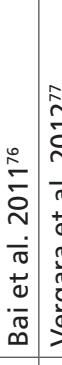 & 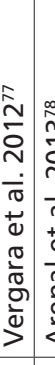 & 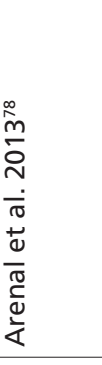 & 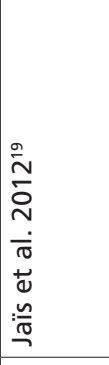 & 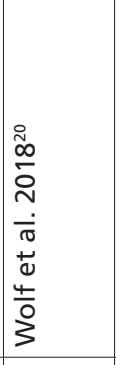 & 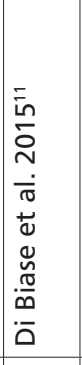 & 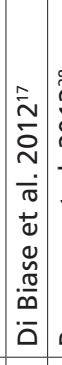 & 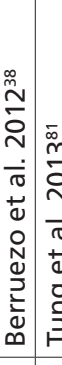 & 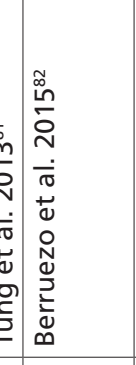 & 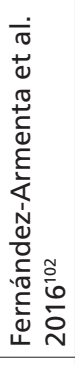 & 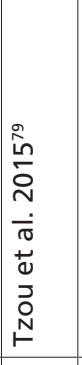 & 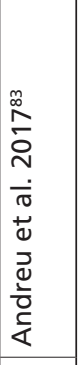 & 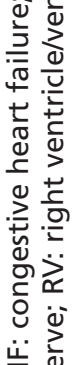 \\
\hline 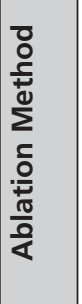 & 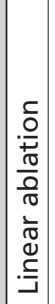 & & $\mid$\begin{tabular}{l|}
$\frac{c}{0}$ \\
$\stackrel{0}{0}$ \\
$\frac{0}{0}$ \\
$\frac{0}{\sigma}$ \\
9 \\
9
\end{tabular} & & & & & & & 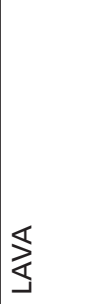 & & 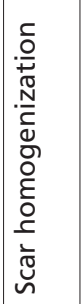 & & 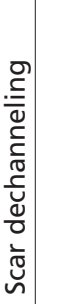 & & & 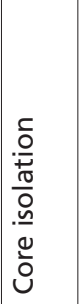 & 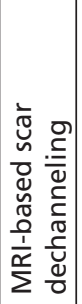 & 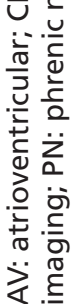 \\
\hline
\end{tabular}



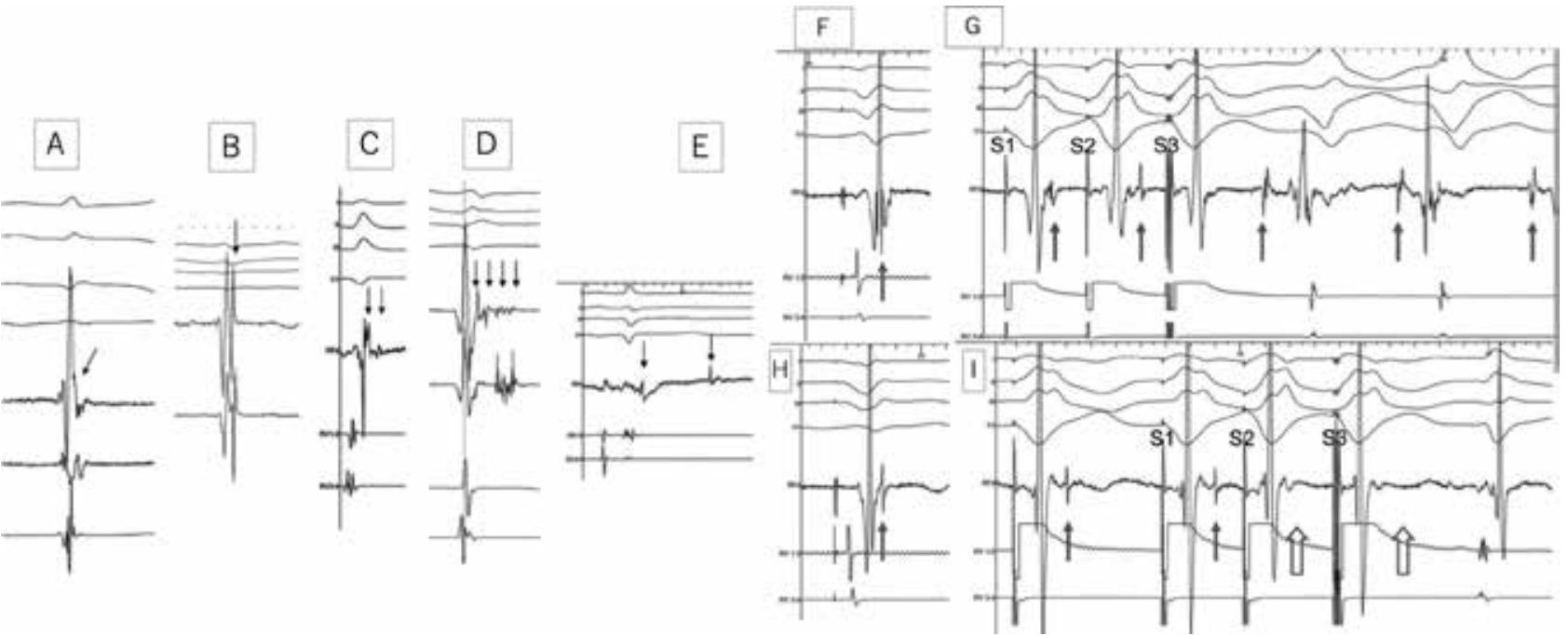

Figure 2: Left: Electrogram recordings from different patients showing characteristics of LAVAs (arrows). A: The potential representing LAVA is fused with the terminal portion of the far-field ventricular signal, making it difficult to identify the LAVA as a separate signal. B: The LAVA potential occurs just after and with a slightly higher frequency than the far-field ventricular potential. LAVAs in A and B occur within the QRS complex. C: The LAVA is a double-component potential that closely follows the far-field ventricular signal. The early component is a high-frequency potential that is almost fused with the preceding far-field ventricular potential. It occurs within the terminal portion of the QRS complex. Another low-amplitude signal follows an isoelectric interval and represents the late component of the LAVA, which occurs after the QRS complex. D: LAVAs are represented by pluricomponent signals without isoelectric intervals. These signals can be visualized distinctly from the preceding far-field ventricular signal. E: A double-component LAVA signal. Although the early component is recorded just after the QRS complex, the late component is recorded after the inscription of the T-wave on the surface electrocardiogram. Right: Role of LAVAs in the induction of VT and the influence of radiofrequency (RF) energy on LAVAs. F: The local ventricular electrogram during the baseline paced rhythm at first sight looks simple. However, in the terminal portion, a very high-frequency component (LAVA) may be identified. G: Programmed electric stimulation from the right ventricle (RV) unmasks the LAVA potential by increasing the delay from the far-field signal. The delay observed during RV pacing suggests poor coupling of the muscle bundle generating the LAVA signal. The delay is maximal with S3, which is associated not only with a change in the polarity of the LAVA but also with the induction of VT. H: After delivery of RF energy, there is a remarkable delay (see A) between the far-field ventricular signal and LAVAs during baseline paced rhythm. I: Repeat programmed electric stimulation from the RV results in the absence of LAVA signals after the far-field ventricular potential during S2 and S3 (open arrows). The absence of LAVAs is associated with an inability to induce VT. Although ablation has rendered the VT noninducible, further RF energy

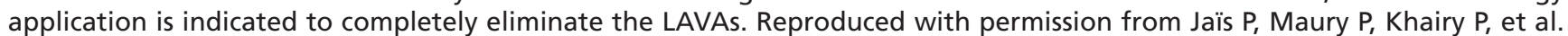
Elimination of local abnormal ventricular activities: a new end point for substrate modification in patients with scar-related ventricular tachycardia. Circulation. 2012;125(18):2184-2196.

Lastly, omnipolar mapping is a new development that may resolve some of these limitations by providing instantaneous catheter-tip wavefront direction and speed. ${ }^{70,71}$ With this mapping technology, local electrical field signals are determined and used to assess the traveling wavefront on a multielectrode catheter, rather than activation-based data acquisition, which may allow for a beat-to-beat determination of wave propagation information that is independent of electrode orientation or activation time. Further clinical validation of this technology is ongoing.

\section{Specific electrogram-based substrate ablation strategies}

Substrate-based ablation approaches may differ between VT ablation centers. A number of methods are implemented during substrate modification, with a large variation in the amount of ablation energy delivered according to the preset mapping and endpoints of the procedure. The major strategies are summarized in Table 1 . These studies must be cautiously interpreted because the mapping details or endpoints are often heterogeneous, even for the same strategy. In addition, although the advantage of multipolar mapping catheters has now been recognized, many previous studies have used ablation catheters for mapping.

\section{Local abnormal ventricular activity-guided ablation.} In a seminal study by our group, we reported a mapping and ablation strategy to homogenize substrate defined as LAVAs (Figure 2). ${ }^{19-21}$ Elimination of all LAVAs is associated with improved midterm and long-term arrhythmia-free survival. ${ }^{19-21}$ LAVAs are defined as sharp, high-frequency ventricular potentials, possibly of low amplitude, that are distinct from the far-field ventricular electrogram that occurs at any time during or after the far-field ventricular electrogram in sinus rhythm or before the far-field ventricular electrogram during VT, which sometimes display 
fractionation or double or multiple components separated by very-low-amplitude signals or an isoelectric interval and which are poorly coupled to the rest of the myocardium. ${ }^{19,21}$ Importantly, this strategy also targets abnormal substrate in so-called normal-voltage areas, although most LAVAs are generally observed in low-voltage areas. ${ }^{53}$ Clinical outcomes have been reported as including a 55\% (88/159) VT freedom rate during a median follow-up of 47 months (range: 33-82 months) without antiarrhythmic drug therapy except for $\beta$-blockers. ${ }^{20}$

Linear ablation with cross-section of the scar and border-zone. Marchlinski et al. ${ }^{10}$ first described the use of linear ablation lesions to target multiple unmappable VTs. The technique involved the creation of contiguous lesions from the dense infarct area through the infarct border-zone and anchored to anatomic barriers or healthy myocardium. In their study, ${ }^{10}$ the ablation strategy resulted in a 75\% (4/16) freedom from VT recurrence rate at the median follow-up point of eight months (range: 3-36 months). Additionally, Soejima et al. ${ }^{72}$ reported a VT freedom rate of $62.5 \%(25 / 40)$ at a mean follow-up point of 12 months \pm six months in their study. In addition, this linear ablation approach was the main approach used in the Substrate Mapping and Ablation in Sinus Rhythm to Halt VT (SMASH VT) trial, ${ }^{22}$ a randomized study showing promising results.

Late-potential ablation. Definitions of LPs differ among studies. ${ }^{16,73-78}$ The initial description was of any electrogram with a duration extending beyond the end of the surface QRS. ${ }^{32}$ Modified definitions have subsequently been reported, often with an isoelectric line among multiple components in the bipolar signals. ${ }^{73,74}$ Regarding the clinical result, Arenal et al. ${ }^{73}$ first reported that after a mean follow-up of nine months \pm four months, no VT recurrence was observed in 19 (79\%) of 24 patients. Volkmer et al. ${ }^{16}$ additionally demonstrated a $71 \%$ VT freedom rate $(7 / 25)$ in a follow-up period of 26 months \pm 14 months. Nogami et al. ${ }^{74}$ reported a $67 \%$ (6/18) VT freedom rate over a relatively long follow-up period of 61 months \pm 38 months in patients with ARVC. Garcia et al. ${ }^{75}$ and Bai et al. ${ }^{76}$ also demonstrated results of the elimination of delayed potentials or LPs in patients with ARVC with follow-up [VT freedom in 77\% $(10 / 13)$ patients during 18 months \pm 13 months of follow-up and ventricular arrhythmia or implantable cardioverter-defibrillator (ICD) appropriate therapy freedom in $84.6 \%$ (22/26) during 39 months \pm four months of follow-up, respectively]. More recently, Vergara et al. ${ }^{77}$ reported that, after a mean follow-up of 13 months \pm four months, VT recurred in 10 patients $(20 \%)$.

Scar homogenization. Di Biase et al. ${ }^{17}$ reported on a scar homogenization approach targeting all abnormal electrograms within low-voltage areas defined with conventional bipolar voltage criteria when mapping in sinus or paced rhythm. With this approach, abnormal electrograms are defined as any electrograms that have more than three deflections, an amplitude of less than $1.5 \mathrm{mV}$, and a duration of more than $70 \mathrm{~ms}$. The acute ablation endpoint was either the elimination of abnormal electrograms or the loss of local capture at high-output pacing (20 mA output at a 10-ms pulse width). This approach can potentially eliminate more possible critical sites than more focused mapping approaches, but has limitations in patients with massive substrate, particularly under unstable conditions. During a mean follow-up of 25 months \pm 10 months, the freedom from VT recurrence rate was $81 \%(35 / 43)$ in patients with ICM who showed scar homogenization. ${ }^{17}$ More recently, the results of a multicenter randomized study comparing scar homogenization with standard limited substrate ablation in patients with ICM were reported. ${ }^{11}$ At one year of follow-up, freedom from VT recurrence was achieved in $52 \%(31 / 60)$ of patients who underwent clinical VT ablation only versus in $85 \%(49 / 58)$ of patients who underwent scar homogenization.

Border-zone ablation/core isolation. The core isolation approach was recently developed by Tzou et al. ${ }^{79}$ in an effort to limit the number of lesions required to eliminate all of the areas critical for VT maintenance within the dense scar. This is a stepwise approach that starts with the identification of the potential critical isthmus within the dense scar that is related to the patient's clinical and/ or induced VTs based on conventional criteria including voltage channels; sites with LPs; sites with good pacemaps; and the existence of long stimulus to QRS intervals, isthmus sites defined by entrainment mapping, and sites of VT termination with ablation. Therefore, this approach acts as a combined approach between conventional and substrate-based approaches. These areas are typically within areas of dense scar $(<0.5 \mathrm{mV})$. Once identified, the critical area is targeted with contiguous ablation lesions either completely surrounding the region of interest or by using anatomic anchors to minimize the amount of ablation necessary. The authors demonstrated that, after a mean follow-up of 18 months \pm nine months, no VT recurrence was observed in $38(86 \%)$ of 44 patients. $^{79}$

Scar dechanneling. This substrate ablation approach, which targets channels within the abnormal substrate, was originally described by Soejima et al. ${ }^{45}$ and Arenal et al. ${ }^{80}$ Although, in all studies, the concept of scar dechanneling encompasses targeting the VT channels, the identification of the channels differs in terms of technique. In the study by Soejima et al., ${ }^{45}$ channels were identified within the low-voltage area using high-output pacing (10 mA, with pulse width of $2 \mathrm{~ms}$ ). Electrically unexcitable scar was defined as a loss of capture at high-output pacing and marked on the voltage maps. Conversely, Arenal et al. ${ }^{80}$ were able to visualize channels after adjusting voltage cutoffs on EAM. More recently, Tung et al. ${ }^{81}$ and Berruezo et al. ${ }^{82}$ described an approach that targets interconnected activation channels within the abnormal substrate, adopting clear endpoints with clinical follow-up. The method involved high-density mapping of the channels of activation of LPs. Once a specific sequence of LP 
activation has been identified, focal ablation of the earliest LP is delivered with the end goal of eliminating a consecutive series of LPs. Tung et al. ${ }^{81}$ demonstrated a rate of freedom from VT recurrence of $86 \%(18 / 21)$ during a median follow-up of 11 months (range: 6-18 months), while Berruezo et al. ${ }^{82}$ noted that, during a median follow-up of 21 months (range: 11-29 months), the rate of freedom of VT recurrence was $80 \%$ (80/101). In addition, Andreu et al. ${ }^{83}$ recently demonstrated scar dechanneling by using CMR imaging in conjunction with EAM, which showed corridors formed by conducting channel points in the scar tissue. In that study, the rate of VT freedom during a mean follow-up of 20 months \pm 19 months was $81.5 \%(44 / 54){ }^{83}$

\section{Frequency analysis mapping}

High-frequency electrogram components are more often associated with critical sites of reentry as compared with low-frequency, large-amplitude components. Several studies have demonstrated that the frequency analysis of electrograms may aid with substrate identification ${ }^{84-86}$; however, this analysis is still only available as an offline tool and the feasibility of an automated real-time tool needs to be further investigated.

\section{Use of imaging to identify substrate}

Cardiac imaging may play an important role in the preprocedural assessment of cardiac anatomy and myocardial scar as well as in the intraprocedural integration of the structural VT substrate. ${ }^{28}$ Cardiac imaging has been mainly used as an adjunct either offline or online (realtime image integration $)^{27,83,87,88}$ to support the localization of scar in 3D mapping systems during substrate mapping and ablation (Figure 3 ). Cardiac imaging has several advantages, as follows: (1) it may provide precise anatomical information including endocardial/ intramural/epicardial scar location, while EAM systems can only provide derived 3D reconstructions from
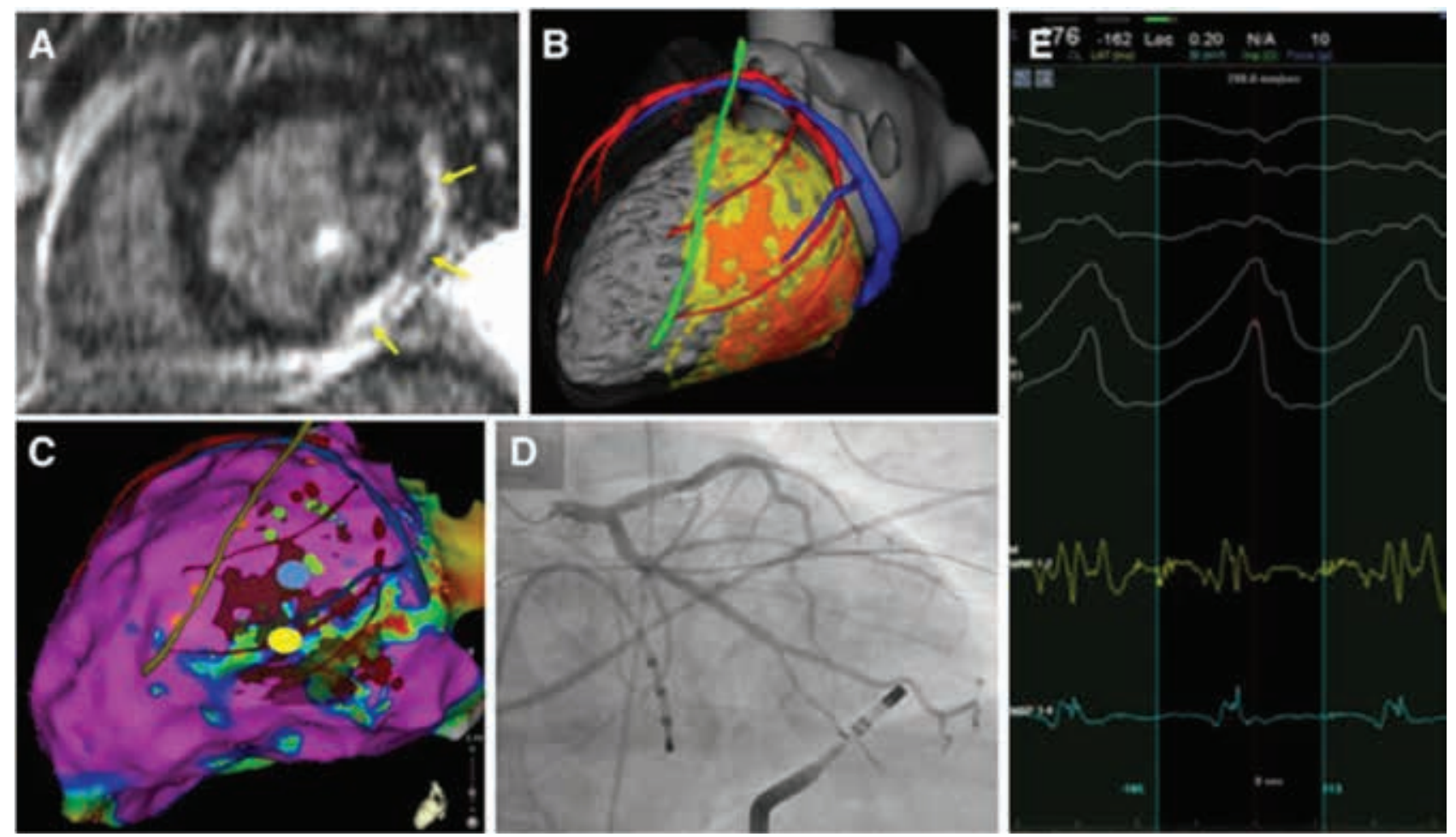

Figure 3: A: Lateral and inferior LV scar on CMR (arrows). B: Patient-specific 3D model built from merged computed tomography (CT) (anatomy) and magnetic resonance imaging (scar) data. Cardiac chambers (gray), coronary arteries, veins (in red and blue, respectively), and left phrenic nerve (green) as segmented from CT and dense scar and gray zone (in orange and yellow, respectively) as segmented from magnetic resonance imaging. C: Epicardial bipolar voltage map with merged imaging model. Voltage mapping (color-coded from purple to red) underestimates the substrate extent as compared with imaging. Fractionated and LPs (green dots) are identified in normal voltage areas. Middiastolic potentials (yellow and blue signals in E) are recorded during VT on an epicardial lateral LV site (yellow dot in C). This potential target for epicardial ablation is far enough from the left phrenic nerve path derived from imaging (green line in C), which accurately matches sites of phrenic capture (orange dots in C). However, CT demonstrates the proximity of this site to a marginal branch of the circumflex artery on the registered imaging model. D: Confirmatory coronary angiography demonstrates contact between the tip of the ablation catheter and the coronary artery. Ablation was thus performed on a different site of the VT isthmus (blue dot in C), resulting in successful VT termination. Reproduced with permission from Mahida S, Sacher F, Dubois R, et al. Cardiac imaging in patients with ventricular tachycardia. Circulation. 2017;136(25):2491-2507. 


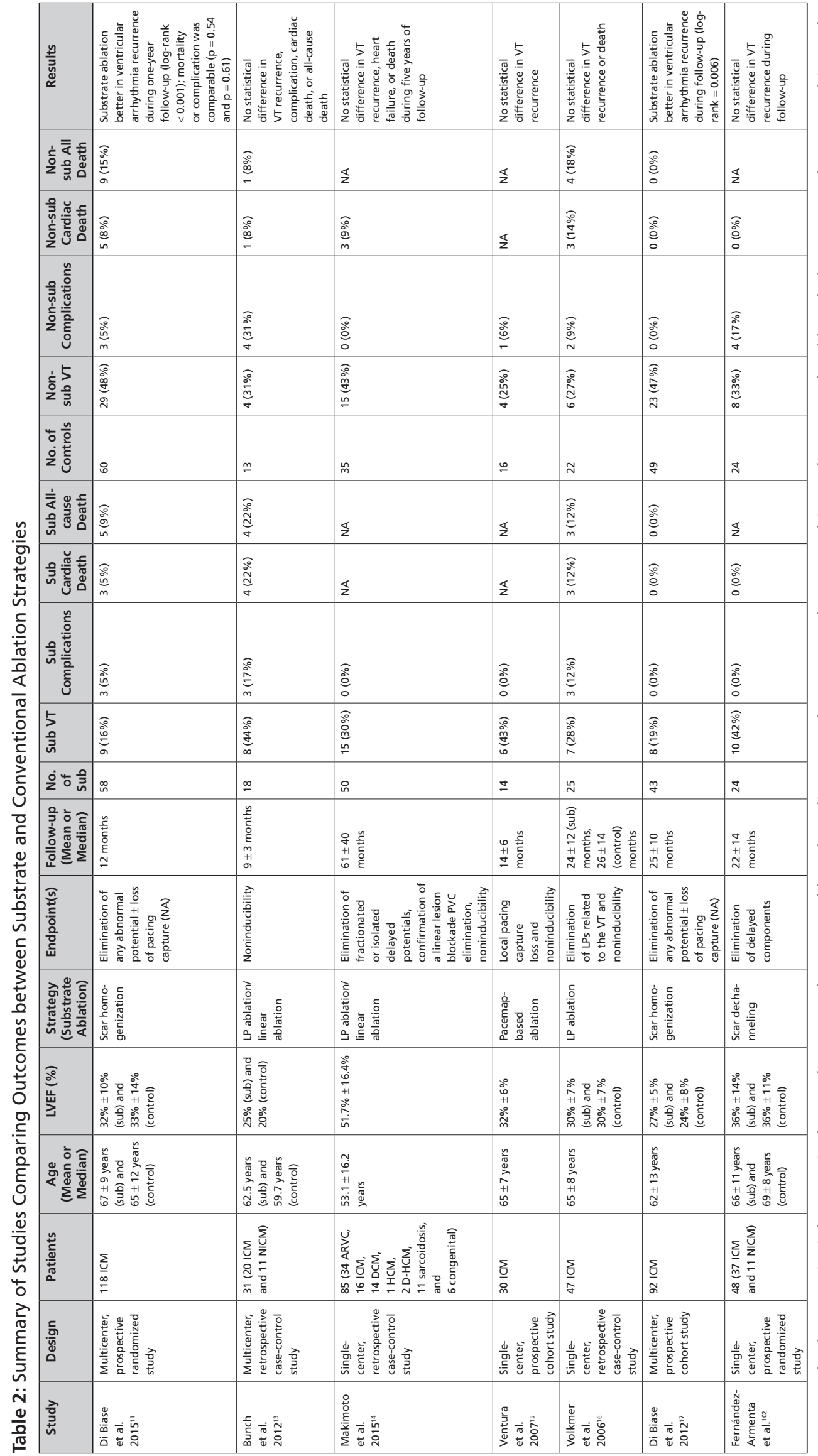

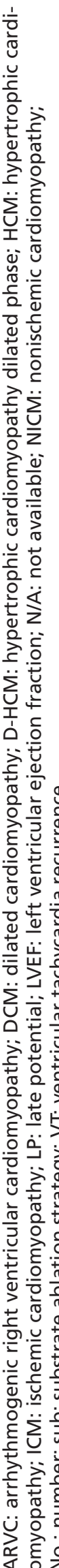


catheter-electrode contact at the myocardial surface; (2) there is no possibility of inaccuracy due to extrapolation, lack of catheter contact, confounding effects of far-field electrograms, or the influence of wavefront activations; (3) it provides information about adjacent anatomical structures, which may affect mapping and ablation, such as papillary muscles, coronary arteries, phrenic nerves, and epicardial fat. However, there are also limitations in imaging techniques in terms of feasibility (eg, magnetic resonance imaging in some patients with old ICDs, MDCT in patients with severe chronic kidney failure), the availability of images with 3D mapping systems, and registration issues.

Recently, in an attempt to refine targeted VT ablation strategies further, several studies have focused on identifying specific scar regions that harbor critical VT isthmuses. ${ }^{29,67,89}$ At this time, scar regions with increased transmurality, scar border zones, and regions at the scar-core-border-zone transition point have been identified as potential targets. ${ }^{29,90}$ CMR has been widely used in this regard, and several studies have shown good correlation with $\mathrm{EAM}^{30,31,62,83,88}$ and a positive clinical impact. ${ }^{27,52,87}$ Further potential benefits of real-time CMR guidance ${ }^{91-93}$ could include improved procedural supervision without exposure to radiation/contact EAM as well as improved substrate detection and lesion visualization according to CMR-defined endpoints. However, CMR may be unavailable, contraindicated, or of suboptimal quality because of ICD-related artifacts, and MDCT represents a valuable alternative for imaging integration. MDCT has been used in combination with EAM to accurately identify dense scar and border-zone regions with significantly higher special resolution ${ }^{29,68}$ as compared with CMR and with high clinical effectiveness. ${ }^{27}$ Studies have mostly included patients with ICM, ${ }^{29}$ while the correlation in patients with NICM is less robust. ${ }^{67,68,87,89}$ A further advantage of MDCT is in the definition of high-resolution cardiac anatomy. ${ }^{47,94} \mathrm{CMR}$ and MDCT may visualize potential isthmuses as VT substrate; however, a certain proportion of circuits are at least partially functional ${ }^{95}$ and the registration needs to be accurate and reproducible.

Other modalities such as intracardiac echocardiography ${ }^{96,97}$ and nuclear imaging ${ }^{98,99}$ may also help to identify substrate. In addition, electrocardiographic imaging incorporated with CMR or MDCT has the potential to identify VT isthmuses noninvasively. ${ }^{100}$ Furthermore, an entirely noninvasive approach that combines anatomical imaging with electrocardiographic imaging and noninvasive cardiac radiotherapy for ablation has been reported, which represents another intriguing strategy that employs cardiac imaging. ${ }^{101}$

\section{Clinical outcomes of substrate-based ablation}

Overall, the clinical outcome of substrate-based ablation is a VT recurrence-free rate of approximately $54 \%$ to $91 \%$ in mid- to long-term follow-up (Table 1). As described above, the success rates, however, vary widely with different strategies and across studies. We have also compared clinical outcomes between substrateand nonsubstrate-based ablation (Table 2). Di Biase et al. ${ }^{11}$ demonstrated a superior VT-free survival rate at 12 months in conjunction with extensive scar homogenization in patients with ICM in a randomized trial [ie, the Ablation of Clinical VT versus Addition of Substrate Ablation on the Long-term Success Rate of VT Ablation (VISTA) trial], while Fernández-Armenta et al. ${ }^{102}$ also conducted a randomized study comparing substrate-based ablation to conventional ablation and demonstrated comparable VT-free survival rates between the two strategies. A recent meta-analysis has also revealed similar acute procedural efficacy, complication, VT recurrence, and mortality rates while comparing a predominantly substrate-based ablation strategy to a strategy guided by activation and entrainment mapping of inducible and hemodynamically tolerated VTs. ${ }^{03}$ A separate meta-analysis ${ }^{104}$ also showed a significantly lower risk of the composite primary outcome of longterm VA recurrence and all-cause mortality among those undergoing substrate modification in comparison with standard ablation in a cohort of mostly patients with ICM. Furthermore, in this study, long-term success was improved when performing complete substrate modification. ${ }^{104}$ Hence, substrate ablation may be superior to a conventional strategy in terms of VT recurrence when extensive substrate ablation is performed. ${ }^{11}$

However, despite the substantial progress that has been made in the use of cardiac imaging to guide VT ablation, there is insufficient evidence at present to suggest that the use of imaging can add value to clinical outcomes. Although observational, nonrandomized studies suggest that image integration may have an impact on procedural outcomes, ${ }^{27,83,87,88}$ well-designed, prospective randomized studies are needed to assess the true impact of image integration as well as to evaluate the potential mechanism(s) of any benefit.

\section{Conclusion}

Several substrate-based ablation strategies have been developed, which include extensive or less-extensive ablation lesions according to the preset mapping and endpoints of the procedure. Although multipolar mapping catheters with smaller and more-narrowly-spaced bipolar electrodes are now widely used, most currently available studies use data acquired by way of ablation catheters. Advances in cardiac imaging may be helpful in providing refined anatomical substrate details.

At this time, clinical outcomes of substrate-based ablation are at least comparable with and possibly superior to conventional VT ablation. The further development of mapping technologies, cardiac imaging, and novel modalities and the incorporation of these modalities in delineating VT substrate may additionally improve the clinical outcomes of substrate-based ablation. 


\section{References}

1. Sapp JL, Wells GA, Parkash R, et al. Ventricular tachycardia ablation versus escalation of antiarrhythmic drugs. $N$ Engl J Med. 2016;375(2):111-121.

2. Santangeli P, Muser D, Maeda S, et al. Comparative effectiveness of antiarrhythmic drugs and catheter ablation for the prevention of recurrent ventricular tachycardia in patients with implantable cardioverter-defibrillators: a systematic review and meta-analysis of randomized controlled trials. Heart Rhythm. 2016;13(7):1552-1559.

3. El-Shalakany A, Hadjis T, Papageorgiou P, Monahan K, Epstein L, Josephson ME. Entrainment/mapping criteria for the prediction of termination of ventricular tachycardia by single radiofrequency lesion in patients with coronary artery disease. Circulation. 1999;99(17):2283-2289.

4. Stevenson WG, Friedman PL, Sager PT, et al. Exploring postinfarction reentrant ventricular tachycardia with entrainment mapping. J Am Coll Cardiol. 1997;29(6):1180-1189.

5. de Chillou C, Lacroix D, Klug D, et al. Isthmus characteristics of reentrant ventricular tachycardia after myocardial infarction. Circulation. 2002;105(6):726-731.

6. Aliot EM, Stevenson WG, Almendral-Garrote JM, et al. EHRA/HRS Expert Consensus on Catheter Ablation of Ventricular Arrhythmias: developed in a partnership with the European Heart Rhythm Association (EHRA), a Registered Branch of the European Society of Cardiology (ESC), and the Heart Rhythm Society (HRS); in collaboration with the American College of Cardiology (ACC) and the American Heart Association (AHA). Heart Rhythm. 2009;6(6):886-933.

7. Stevenson WG, Friedman PL, Kocovic D, Sager PT, Saxon LA, Pavri B. Radiofrequency catheter ablation of ventricular tachycardia after myocardial infarction. Circulation. 1998;98(4):308-314.

8. Callans DJ, Zado E, Sarter BH, Schwartzman D, Gottlieb CD, Marchlinski FE. Efficacy of radiofrequency catheter ablation for ventricular tachycardia in healed myocardial infarction. Am J Cardiol. 1998;82(4):429-432.

9. Cassidy DM, Vassallo JA, Marchlinski FE, Buxton AE, Untereker WJ, Josephson ME. Endocardial mapping in humans in sinus rhythm with normal left ventricles: Activation patterns and characteristics of electrograms. Circulation. 1984;70(1):37-42.

10. Marchlinski FE, Callans DJ, Gottlieb CD, Zado ES. Linear ablation lesions for control of unmappable ventricular tachycardia in patients with ischemic and nonischemic cardiomyopathy. Circulation. 2000;101(11):1288-1296.

11. Di Biase L, Burkhardt JD, Lakkireddy D, et al. Ablation of stable VTs versus substrate ablation in ischemic cardiomyopathy: the VISTA randomized multicenter trial. J Am Coll Cardiol. 2015;66(25):2872-2882.

12. Dello Russo A, Casella M, Pieroni M, et al. Drug-refractory ventricular tachycardias after myocarditis: Endocardial and epicardial radiofrequency catheter ablation. Circ Arrhythmia Electrophysiol. 2012;5(3):492-498.

13. Bunch TJ, Darby A, May HT, et al. Efficacy and safety of ventricular tachycardia ablation with mechanical circulatory support compared with substrate-based ablation techniques. Europace. 2012;14(5):709-714.

14. Makimoto H, Nakajima I, Miyamoto K, et al. Clinical impact of mapping strategies for treatment of ventricular tachycardias in patients with structural heart disease. Pacing Clin Electrophysiol. 2015;38(5):630-640.

15. Ventura R, Klemm HU, Rostock T, et al. Stable and unstable ventricular tachycardias in patients with previous myocardial infarction: A clinically oriented strategy for catheter ablation. Cardiology. 2007;109(1):52-61.
16. Volkmer M, Ouyang F, Deger F, et al. Substrate mapping vs. tachycardia mapping using CARTO in patients with coronary artery disease and ventricular tachycardia: Impact on outcome of catheter ablation. Europace. 2006;8(11):968-976.

17. Di Biase L, Santangeli P, Burkhardt DJ, et al. Endo-epicardial homogenization of the scar versus limited substrate ablation for the treatment of electrical storms in patients with ischemic cardiomyopathy. J Am Coll Cardiol. 2012;60(2):132-141.

18. Gökoğlan Y, Mohanty S, Gianni C, et al. Scar homogenization versus limited-substrate ablation in patients with nonischemic cardiomyopathy and ventricular tachycardia. J Am Coll Cardiol. 2016;68(18):1990-1998.

19. Jaïs P, Maury P, Khairy P, et al. Elimination of local abnormal ventricular activities: A new end point for substrate modification in patients with scar-related ventricular tachycardia. Circulation. 2012;125(18):2184-2196.

20. Wolf M, Sacher F, Cochet H, et al. Long-term outcome of substrate modification in ablation of post-myocardial infarction ventricular tachycardia. Circ Arrhythmia Electrophysiol. 2018;11(2):e005635.

21. Sacher F, Lim HS, Derval N, et al. Substrate mapping and ablation for ventricular tachycardia: the LAVA approach. $J$ Cardiovasc Electrophysiol. 2015;26(4):464-471.

22. Reddy VY, Reynolds MR, Neuzil P, et al. Prophylactic catheter ablation for the prevention of defibrillator therapy. $\mathrm{N}$ Engl J Med. 2007;357(26):2657-2665.

23. Gepstein L, Hayam G, Ben-haim SA. A novel method for nonfluoroscopic catheter-based electroanatomical mapping of the heart. In vitro and in vivo accuracy results. Circulation. 1997;95(6):1611-1622.

24. Berte B, Relan J, Sacher F, et al. Impact of electrode type on mapping of scar-related VT. J Cardiovasc Electrophysiol. 2015;26(11):1213-1223.

25. Anter E, Tschabrunn CM, Josephson ME. High-resolution mapping of scar-related atrial arrhythmias using smaller electrodes with closer interelectrode spacing. Circ Arrhythmia Electrophysiol. 2015;8(3):537-545.

26. Tschabrunn CM, Roujol S, Dorman NC, Nezafat R, Josephson $\mathrm{ME}$, Anter E. High-resolution mapping of ventricular scar: comparison between single and multielectrode catheters. Circ Arrhythmia Electrophysiol. 2016;9(6). pii: e003841.

27. Yamashita S, Cochet H, Sacher F, et al. Impact of new technologies and approaches for post-myocardial infarction ventricular tachycardia ablation during long-term follow-up. Circ Arrhythmia Electrophysiol. 2016;9(7):1-12.

28. Mahida S, Sacher F, Dubois R, et al. Cardiac imaging in patients with ventricular tachycardia. Circulation. 2017;136(25):2491-2507.

29. Yamashita S, Sacher F, Hooks DA, et al. Myocardial wall thinning predicts transmural substrate in patients with scar-related ventricular tachycardia. Heart Rhythm. 2017;14(2):155-163.

30. Wijnmaalen AP, Van Der Geest RJ, Van Huls Van Taxis $\mathrm{CFB}$, et al. Head-to-head comparison of contrast-enhanced magnetic resonance imaging and electroanatomical voltage mapping to assess post-infarct scar characteristics in patients with ventricular tachycardias: real-time image integration and reversed registration. Eur Heart J. 2011;32(1):104-114.

31. Fernández-Armenta J, Berruezo A, Andreu D, et al. Threedimensional architecture of scar and conducting channels based on high resolution ce-CMR: Insights for ventricular tachycardia ablation. Circ Arrhythmia Electrophysiol. 2013;6(3):528-537.

32. Cassidy DM, Vassallo JA, Miller JM, et al. Endocardial catheter mapping in patients in sinus rhythm: Relationship to underlying heart disease and ventricular arrhythmias. Circulation. 1986;73(4):645-652. 
33. Cassidy DM, Vassallo JA, Buxton AE, Doherty JU, Marchlinski FE, Josephson ME. The value of catheter mapping during sinus rhythm to localize site of origin of ventricular tachycardia. Circulation. 1984;69(6):1103-1110.

34. Kienzle MG, Miller J, Falcone RA, Harken A, Josephson ME. Intraoperative endocardial mapping during sinus rhythm: Relationship to site of origin of ventricular tachycardia. Circulation. 1984;70(6):957-965.

35. Acosta J, Penela D, Andreu D, et al. Multielectrode vs. pointby-point mapping for ventricular tachycardia substrate ablation: a randomized study. Europace. 2018;20(3):512-519.

36. Sacher F, Duchateau J, Capellino S, et al. Voltage threshold for high density mapping catheter with short interspersed small electrodes. Heart Rhythm. 2016;13:S32.

37. Maagh P, Christoph A, Dopp H, Mueller MS, Plehn G, Meissner A. High-density mapping in ventricular tachycardia ablation: a PentaRay ${ }^{\circledR}$ study. Cardiol Res. 2017;8(6):293-303.

38. Berruezo A, Fernández-Armenta J, Mont L, et al. Combined endocardial and epicardial catheter ablation in arrhythmogenic right ventricular dysplasia incorporating scar dechanneling technique. Circ Arrhythmia Electrophysiol. 2012;5(1):111-121.

39. Haqqani HM, Kalman JM, Roberts-Thomson KC, et al. Fundamental differences in electrophysiologic and electroanatomic substrate between ischemic cardiomyopathy patients with and without clinical ventricular tachycardia. J Am Coll Cardiol. 2009;54(2):166-173.

40. Kumar S, Baldinger SH, Kapur S, et al. Right ventricular scar-related ventricular tachycardia in nonischemic cardiomyopathy: electrophysiological characteristics, mapping, and ablation of underlying heart disease. J Cardiovasc Electrophysiol. 2018;29(1):79-89.

41. Deneke T, Müller KM, Lemke B, et al. Human histopathology of electroanatomic mapping after cooled-tip radiofrequency ablation to treat ventricular tachycardia in remote myocardial infarction. I Cardiovasc Electrophysiol. 2005;16(11):1246-1251.

42. Koa-Wing M, Ho SY, Kojodjojo P, Peters NS, Davies DW, Kanagaratnam P. Radiofrequency ablation of infarct scar-related ventricular tachycardia: correlation of electroanatomical data with post-mortem histology. J Cardiovasc Electrophysiol. 2007;18(12):1330-1333.

43. Codreanu A, Odille F, Aliot E, et al. Electroanatomic characterization of post-infarct scars comparison with 3-dimensional myocardial scar reconstruction based on magnetic resonance imaging. J Am Coll Cardiol. 2008;52(10): 839-842.

44. Anter E, Tschabrunn C, Rajoul S. In vivo high-resolution mapping of post-infarction reentrant ventricular tachycardias. Heart Rhythm. 2015;12:S203.

45. Soejima K, Stevenson WG, Maisel WH, Sapp JL, Epstein LM. Electrically unexcitable scar mapping based on pacing threshold for identification of the reentry circuit isthmus: feasibility for guiding ventricular tachycardia ablation. Circulation. 2002;106(13):1678-1683.

46. Cano O, Hutchinson M, Lin D, et al. Electroanatomic substrate and ablation outcome for suspected epicardial ventricular tachycardia in left ventricular nonischemic cardiomyopathy. J Am Coll Cardiol. 2009;54(9):799-808.

47. Desjardins B, Morady F, Bogun F. Effect of epicardial fat on electroanatomical mapping and epicardial catheter ablation. J Am Coll Cardiol. 2010;56(16):1320-1327.

48. Verma A, Marrouche NF, Schweikert RA, et al. Relationship between successful ablation sites and the scar border zone defined by substrate mapping for ventricular tachycardia post-myocardial infarction. J Cardiovasc Electrophysiol. 2005;16(5):465-471.
49. De Bakker JMT, Van Capelle FJL, Janse MJ, et al. Slow conduction in the infarcted human heart: 'zigzag' course of activation. Circulation. 1993;88(3):915-926.

50. Kettering K, Weig HJ, Reimold M, et al. Catheter ablation of ventricular tachycardias in patients with ischemic cardiomyopathy: Validation of voltage mapping criteria for substrate modification by myocardial viability assessment using FDG PET. Clin Res Cardiol. 2010;99(11):753-760.

51. de Riva M, Naruse $Y$, Ebert M, et al. Targeting the hidden substrate unmasked by right ventricular extrastimulation improves ventricular tachycardia ablation outcome after myocardial infarction. JACC Clin Electrophysiol. 2018;4(3):316-327.

52. Porta-Sánchez A, Jackson N, Lukac P, et al. Multicenter study of ischemic ventricular tachycardia ablation with decrement evoked potential (DEEP) mapping with extra stimulus. JACC Clin Electrophysiol. 2018;4(3):307-315.

53. Komatsu Y, Daly M, Sacher F, et al. Electrophysiologic characterization of local abnormal ventricular activities in postinfarction ventricular tachycardia with respect to their anatomic location. Heart Rhythm. 2013;10(11):1630-1637.

54. Tung R, Josephson ME, Bradfield JS, Shivkumar K. Directional influences of ventricular activation on myocardial scar characterization: voltage mapping with multiple wavefronts during ventricular tachycardia ablation. Circ Arrhythmia Electrophysiol. 2016;9(8). pii: e004155.

55. Josephson ME, Anter E. Substrate mapping for ventricular tachycardia assumptions and misconceptions. JACC Clin Electrophysiol. 2015;1(5):341-352.

56. Roberts DE, Hersh LT, Scher AM. Influence of cardiac fiber orientation on wavefront voltage, conduction velocity, and tissue resistivity in the dog. Circ Res. 1979;44(5):701-712.

57. Hsia HH, Callans DJ, Marchlinski FE. Characterization of endocardial electrophysiological substrate in patients with nonischemic cardiomyopathy and monomorphic ventricular tachycardia. Circulation. 2003;108(6):704-710.

58. Haqqani HM, Tschabrunn CM, Tzou WS, et al. Isolated septal substrate for ventricular tachycardia in nonischemic dilated cardiomyopathy: Incidence, characterization, and implications. Heart Rhythm. 2011;8(8):1169-1176.

59. Polin GM, Haqqani H, Tzou W, et al. Endocardial unipolar voltage mapping to identify epicardial substrate in arrhythmogenic right ventricular cardiomyopathy/dysplasia. Heart Rhythm. 2011;8(1):76-83.

60. Hutchinson MD, Gerstenfeld EP, Desjardins B, et al. Endocardial unipolar voltage mapping to detect epicardial ventricular tachycardia substrate in patients with nonischemic left ventricular cardiomyopathy. Circ Arrhythmia Electrophysiol. 2011;4(1):49-55.

61. Campos B, Jauregui ME, Park KM, et al. New unipolar electrogram criteria to identify irreversibility of nonischemic left ventricular cardiomyopathy. J Am Coll Cardiol. 2012;60(21):2194-2204.

62. Desjardins B, Crawford T, Good E, et al. Infarct architecture and characteristics on delayed enhanced magnetic resonance imaging and electroanatomic mapping in patients with postinfarction ventricular arrhythmia. Heart Rhythm. 2009;6(5):644-651.

63. Duchateau J, Dubois R. High-density mapping of atrial tachycardias: importance of interpolation. I Cardiovasc Electrophysiol. 2018;29(5):E7-E8.

64. Blauer JJE, Swenson D, Higuchi K, et al. Sensitivity and specificity of substrate mapping: an in silico framework for the evaluation of electroanatomical substrate mapping strategies. J Cardiovasc Electrophysiol. 2014;25(7):774-780.

65. Teijeira-Fernandez E, Cochet H, Bourier F, et al. Influence of contact force on voltage mapping: a combined magnetic 
resonance imaging and electroanatomic mapping study in patients with tetralogy of Fallot. Heart Rhythm. 2018;15(8):1198-1205.

66. Mizuno H, Vergara P, MacCabelli G, et al. Contact force monitoring for cardiac mapping in patients with ventricular tachycardia. J Cardiovasc Electrophysiol. 2013;24(5): 519-524.

67. Esposito A, Palmisano A, Antunes S, et al. Cardiac CT with delayed enhancement in the characterization of ventricular tachycardia structural substrate: relationship between CT-segmented scar and electro-anatomic mapping. JACC Cardiovasc Imaging. 2016;9(7):822-832.

68. Tian J, Jeudy J, Smith MF, et al. Three-dimensional contrast-enhanced multidetector CT for anatomic, dynamic, and perfusion characterization of abnormal myocardium to guide ventricular tachycardia ablations. Circ Arrhythmia Electrophysiol. 2010;3(5):496-504.

69. Berruezo A, Paetsch I. Inception: implanting the idea of magnetic resonance imaging-guided ventricular tachycardia substrate ablation. Europace. 2018;20(FI2):f143-f145.

70. Massé S, Magtibay K, Jackson N, et al. Resolving myocardial activation with novel omnipolar electrograms. Circ Arrhythmia Electrophysiol. 2016;9(7):e004107.

71. Deno DC, Balachandran R, Morgan D, Ahmad F, Masse S, Nanthakumar K. Orientation-independent catheter-based characterization of myocardial activation. IEEE Trans Biomed Eng. 2017;64(5):1067-1077.

72. Soejima K, Suzuki M, Maisel WH, et al. Catheter ablation in patients with multiple and unstable ventricular tachycardias after myocardial infarction: short ablation lines guided by reentry circuit isthmuses and sinus rhythm mapping. Circulation. 2001;104(6):664-669.

73. Arenal A, Glez-Torrecilla E, Ortiz M, et al. Ablation of electrograms with an isolated, delayed component as treatment of unmappable monomorphic ventricular tachycardias in patients with structural heart disease. J Am Coll Cardiol. 2003;41(1):81-92.

74. Nogami A, Sugiyasu A, Tada H, et al. Changes in the isolated delayed component as an endpoint of catheter ablation in arrhythmogenic right ventricular cardiomyopathy: predictor for long-term success. J Cardiovasc Electrophysiol. 2008;19(7):681-688.

75. Garcia FC, Bazan V, Zado ES, Ren JF, Marchlinski FE. Epicardial substrate and outcome with epicardial ablation of ventricular tachycardia in arrhythmogenic right ventricular cardiomyopathy/dysplasia. Circulation. 2009;120(5):366-375.

76. Bai R, Di Biase L, Shivkumar K, et al. Ablation of ventricular arrhythmias in arrhythmogenic right ventricular dysplasia/cardiomyopathy: arrhythmia-free survival after endo-epicardial substrate based mapping and ablation. Circ Arrhythmia Electrophysiol. 2011;4(4):478-485.

77. Vergara P, Trevisi N, Ricco A, et al. Late potentials abolition as an additional technique for reduction of arrhythmia recurrence in scar related ventricular tachycardia ablation. $J$ Cardiovasc Electrophysiol. 2012;23(6):621-627.

78. Arenal Á, Hernández J, Calvo D, et al. Safety, long-term results, and predictors of recurrence after complete endocardial ventricular tachycardia substrate ablation in patients with previous myocardial infarction. Am J Cardiol. 2013;111(4):499-505.

79. Tzou WS, Frankel DS, Hegeman T, et al. Core isolation of critical arrhythmia elements for treatment of multiple scar-based ventricular tachycardias. Circ Arrhythmia Electrophysiol. 2015;8(2):353-361.

80. Arenal A, Del Castillo S, Gonzalez-Torrecilla E, et al. Tachycardia-related channel in the scar tissue in patients with sustained monomorphic ventricular tachycardias: influence of the voltage scar definition. Circulation. 2004;110(17):2568-2574.

81. Tung R, Mathuria NS, Nagel R, et al. Impact of local ablation on interconnected channels within ventricular scar: mechanistic implications for substrate modification. Circ Arrhythmia Electrophysiol. 2013;6(6):1131-1138.

82. Berruezo A, Fernández-Armenta J, Andreu D, et al. Scar dechanneling: new method for scar-related left ventricular tachycardia substrate ablation. Circ Arrhythmia Electrophysiol. 2015;8(2):326-336.

83. Andreu D, Penela D, Acosta J, et al. Cardiac magnetic resonance-aided scar dechanneling: Influence on acute and long-term outcomes. Heart Rhythm. 2017;14(8):1121-1128.

84. Kuroki K, Nogami A, Igarashi M, etal. New substrate-guided method of predicting slow conducting isthmuses of ventricular tachycardia: preliminary analysis to the combined use of voltage limit adjustment and fast-fourier transform analysis. Circ Arrhythmia Electrophysiol. 2018;11(4): e005705.

85. Lin CY, Silberbauer J, Lin YJ, et al. Simultaneous Amplitude Frequency Electrogram Transformation (SAFE-T) mapping to identify ventricular tachycardia arrhythmogenic potentials in sinus rhythm. JACC Clin Electrophysiol. 2016;2(4):459-470.

86. Campos B, Jauregui ME, Marchlinski FE, Dixit S, Gerstenfeld EP. Use of a novel fragmentation map to identify the substrate for ventricular tachycardia in postinfarction cardiomyopathy. Heart Rhythm. 2015;12(1):95-103.

87. Yamashita S, Sacher F, Mahida S, et al. Image integration to guide catheter ablation in scar-related ventricular tachycardia. J Cardiovasc Electrophysiol. 2016;27(6):699-708.

88. Bogun FM, Desjardins B, Good E, et al. Delayed-enhanced magnetic resonance imaging in nonischemic cardiomyopathy. Utility for identifying the ventricular arrhythmia substrate. I Am Coll Cardiol. 2009;53(13):1138-1145.

89. Komatsu Y, Cochet H, Jadidi A, et al. Regional myocardial wall thinning at multidetector computed tomography correlates to arrhythmogenic substrate in postinfarction ventricular tachycardia: Assessment of structural and electrical substrate. Circ Arrhythmia Electrophysiol. 2013;6(2):342-350.

90. Piers SRD, Tao Q, De Riva Silva M, et al. CMR-based identification of critical isthmus sites of ischemic and nonischemic ventricular tachycardia. JACC Cardiovasc Imaging. 2014;7(8):774-784.

91. Grothoff M, Gutberlet M, Hindricks G, et al. Magnetic resonance imaging guided transatrial electrophysiological studies in swine using active catheter tracking - experience with 14 cases. Eur Radiol. 2017;27(5):1954-1962.

92. Sommer P, Grothoff M, Eitel C, et al. Feasibility of real-time magnetic resonance imaging-guided electrophysiology studies in humans. Europace. 2013;15(1):101-108.

93. Dukkipati SR, Mallozzi R, Schmidt EJ, et al. Electroanatomic mapping of the left ventricle in a porcine model of chronic myocardial infarction with magnetic resonance-based catheter tracking. Circulation. 2008;118(8):853-862.

94. Yamashita S, Sacher F, Mahida S, et al. Role of high-resolution image integration to visualize left phrenic nerve and coronary arteries during epicardial ventricular tachycardia ablation. Circ Arrhythmia Electrophysiol. 2015;8(2):371-380.

95. Anter E, Tschabrunn CM, Buxton AE, Josephson ME. Highresolution mapping of postinfarction reentrant ventricular tachycardia: electrophysiological characterization of the circuit. Circulation. 2016;134(4):314-327.

96. Khaykin Y, Skanes A, Whaley B, et al. Real-time integration of 2D intracardiac echocardiography and 3D electroanatomical mapping to guide ventricular tachycardia ablation. Heart Rhythm. 2008;5(10):1396-1402. 
97. Bunch TJ, Weiss JP, Crandall BG, et al. Image integration using intracardiac ultrasound and $3 \mathrm{D}$ reconstruction for scar mapping and ablation of ventricular tachycardia. $J$ Cardiovasc Electrophysiol. 2010;21(6):678-684.

98. Dickfeld T, Lei P, Dilsizian V, et al. Integration of three-dimensional scar maps for ventricular tachycardia ablation with positron emission tomography-computed tomography. JACC Cardiovasc Imaging. 2008;1(1):73-82.

99. Klein T, Abdulghani M, Smith M, et al. Three-dimensional 123I-meta-iodobenzylguanidine cardiac innervation maps to assess substrate and successful ablation sites for ventricular tachycardia: feasibility study for a novel paradigm of innervation imaging. Circ Arrhythmia Electrophysiol. 2015;8(3):583-591.

100. Cochet H, Dubois R, Sacher F, et al. Cardiac arrythmias: multimodal assessment integrating body surface ECG mapping into cardiac imaging. Radiology. 2014;271(1):239-247.
101. Cuculich PS, Schill MR, Kashani R, et al. Noninvasive cardiac radiation for ablation of ventricular tachycardia. $N$ Engl J Med. 2017;377(24):2325-2336.

102. Fernández-Armenta J, Penela D, Acosta J, et al. Substrate modification or ventricular tachycardia induction, mapping, and ablation as the first step? A randomized study. Heart Rhythm. 2016;13(8):1589-1595.

103. Kumar S, Baldinger SH, Romero J, et al. Substrate-based ablation versus ablation guided by activation and entrainment mapping for ventricular tachycardia: a systematic review and meta-analysis. I Cardiovasc Electrophysiol. 2016;27(12):1437-1447.

104. Briceño DF, Romero J, Villablanca PA, et al. Long-term outcomes of different ablation strategies for ventricular tachycardia in patients with structural heart disease: systematic review and meta-analysis. Europace. 2018;20(1): 104-115. 